\title{
Towards a pharmacological neuro-protectant: can anesthesia deliver?
}

\author{
Dermot R. Doherty, MBBCh
}

Published online: 30 July 2009

(c) Canadian Anesthesiologists' Society 2009

Transient global cerebral ischemic injury (tGCI) continues to be a significant cause of death and disability. Global cerebral ischemia usually results from complex medical conditions, including cardiac arrest, circulatory shock, and birth asphyxia or iatrogenic interventions, such as circulatory arrest for cardiovascular surgery. The pathophysiology of global cerebral ischemia is complex. Experimental studies have identified a number of potential therapeutic treatments. Some of these treatments have targeted the initial metabolic events following brain ischemia, including energy failure, calcium ion flux, free radical generation, and the attenuation of excitotoxicity. These harmful mechanisms have been targeted with pharmacological interventions in experimental models. However, none of these promising pharmacological therapies have been successfully translated into clinical use. Such "easy to administer" therapies could be stocked in crash carts for administration to patients once the heart has been restarted. By contrast, physiological therapies, such as therapeutic hypothermia, have gained success in both experimental and clinical settings. ${ }^{1}$ This type of therapy is difficult to administer and involves extensive infrastructure, resources, and personnel. However, even therapeutic hypothermia may not be applicable across all human populations. ${ }^{2}$

Pre-conditioning is a strategy that involves increasing the brain's tolerance to ischemia by exposing the brain to minor ischemic insults or pharmacological agents prior to the occurrence of a major ischemic event. While anesthetic preconditioning has shown initial promise in protecting the brain from ischemic events in pre-clinical studies, to date,

D. R. Doherty, MBBCh $(\square)$

Department of Anesthesiology, Children's Hospital of Eastern Ontario, 401 Smyth Road, Ottawa, ON K1H 8L1, Canada

e-mail: ddoherty@cheo.on.ca there is no convincing clinical epidemiology supporting this practice. ${ }^{3}$ The pre-clinical science regarding the potential of anesthetic preconditioning is far from clear. This is illustrated in a recent article by Codaccioni et al., who report a transient but non-sustained improvement in neurocognitive testing in a rodent model of focal brain ischemia. In reality, this may simply be reflective of and/or specific to the particular model employed in their experiments. Any potential neuroprotective effects of anesthetic preconditioning depend on the anesthetic agent used to precondition, the species studied, the model of ischemia, the age and sex of the animal, the duration of preconditioning, and the interval between preconditioning and ischemic insult. ${ }^{3}$ In other words, it is not clear whether this will translate into a universally applicable therapy for tGCI.

Anesthetic preconditioning is one of several strategies that prepare the brain to anticipate or react to the ischemic insult. Examples of other strategies used to precondition the brain include toxins, temperature, pharmaceuticals, and ischemia itself, with the latter showing most promise. ${ }^{4,5}$ The common theme of these strategies is to unbalance several key cell signal transcriptional programs in favour of cell survival, rather than any single metabolic pathway or target protein.

Neuronal death after tGCI is complex. Characteristic lesions in vulnerable regions of the brain (such as the hippocampus and the striatum) appear from $12 \mathrm{~h}$ to 3 days after resuscitation and continue to evolve in the following weeks to months. Most cells that die do so by programmed cell death (PCD), as opposed to necrosis. This type of neuronal death has several features of apoptosis, with the cysteine-dependent aspartate-directed proteases family (the caspases) featuring strongly as executioners of PCD. ${ }^{6}$ Caspases normally exist in an inactivated state (pro-caspases), but under stressful conditions, cleavage (activation) 
of the initiator-caspases (caspase-8 and 9) takes place. Initiator caspases can be activated by stimuli extrinsic or intrinsic to the cell. For example, when circulating cytokines, such as IL- $1 \beta$ or TNF- $\alpha$, are released after reperfusion and bind to transmembrane protein complexes called "death domains", caspase- 8 cleavage occurs. ${ }^{7}$ Intrinsically, cellular stress from reactive oxygen species or calcium ion flux can cause the activation of protein cascades which destabilize the mitochondrial membrane leading to cleavage of caspase-9. When cleaved, initiator caspases facilitate cleavage of effector caspases (caspase3 ), which proteolyse strategic cellular substrates leading to controlled death of the cell.

This simplistic explanation of cell death after ischemia does not do justice to the spectrum of programmed cell death observed or to the multiple layers of metabolic control, alternative pathways, and complex protein signalling that occurs between the cell membrane, the mitochondrial membranes, and the nucleus via cell death proteins. Many key pro-apoptotic proteins, such as caspases and apoptosis-inducing factor have dual roles important to the normal function of the cell. Consequently, for any given neuroprotective intervention to work, there needs to be sustained unbalancing of signal transductional and transcriptional programs that favour neuronal survival. Also, it should not produce an unintended consequence such as the development of cancer.

Anesthetics agents have been proposed as neuroprotective agents because of their ability to reduce cerebral metabolic rate. On the other hand, intravenous, local, and volatile anesthetics prevent oxidative phosphorylation and ATP synthesis in mitochondria by reversibly altering the structure and/or function of the elements of the inner mitochondrial membrane. ${ }^{8}$ While, at face value, this appears to be an energy-neutral state, the inability to produce scavengers of reactive oxygen species or to regulate intracellular calcium homeostasis may tip the balance in favour of cell death. ${ }^{9}$ Additionally, the substantial body of recent literature reporting that anesthetic agents induce apoptosis in developing brains ${ }^{10}$ and possibly aging brains ${ }^{11}$ may result in a seachange in how we view the tools of our specialty. In future, we may choose our neuroanesthetic agents from the perspective of "least-harm" rather than "prevent-harm". That being said, we are probably at the tip of the iceberg in terms of our understanding of the molecular interactions of anesthesia with cellular metabolic processes in the context of co-existing disease or altered physiology. A clear neuroprotective role has not yet emerged.

Recently, tGCI has been considered a clinical and therapeutic "black box" for several reasons. First, patient-specific factors (e.g., age, sex, co-morbidity) and the underlying pathophysiologic cause of the brain ischemia and its treatment render it difficult to estimate the severity of brain injury. Second, prior to the availability of diffusion MRI and MR-spectroscopy, it was difficult for clinicians to make the connection between the ischemic event and the subsequent development of characteristic brain lesions. Third, no specific pharmacological treatment options are available other than supportive care. These limitations provided a strong barrier to progress until the potential of therapeutic hypothermia was reported. We are now faced with streamlined medical emergency teams for in-hospital physiological decline, a fund of research relating to resuscitation efficacy, and occasionally the option of extra-corporeal circulatory support for cardiorespiratory arrest. This brings greater urgency to a post-resuscitation adjunctive or alternative treatment to therapeutic hypothermia in the neurological care of patients who have suffered tGCI.

In future, strategies that induce a pre-conditioning (protective) response may be utilized to reduce the magnitude of brain injury associated with global ischemia (i.e., pre-circulatory arrest). Experimental data demonstrate that short noxious stimuli optimally timed around an index ischemic event result in up-regulation of "potentially protective" signal transduction programs, including the Akt/P13-K pathway, the mitogen activated protein kinases, ATP-sensitive potassium channels, and the NF- $\kappa$ B pathway. ${ }^{12}$ The promise that these investigations may ultimately transform into the aforementioned intravenous neuro-protectant in the near future is unlikely. Continuing research into this field will help us narrow down the key mechanisms and patientspecific contexts relating to neuronal survival after tGCI. In time, we should have a "best-fit" intervention.

\section{Vers un neuroprotecteur pharmacologique: l'anesthésie peut-elle répondre à la demande?}

L'ischémie cérébrale globale transitoire (tGCI) demeure une importante cause de décès et de morbidité. En règle générale, l'ischémie globale est le résultat de conditions médicales complexes, notamment l'arrêt cardiaque, le choc circulatoire, la mort apparente du nouveau-né et les interventions iatrogéniques telles que l'arrêt circulatoire pour une chirurgie cardiovasculaire. La physiopathologie de l'ischémie globale est elle aussi complexe. Des études expérimentales ont identifié plusieurs traitements thérapeutiques potentiels; certains de ces traitements ciblent les événements métaboliques initiaux suivant l'ischémie cérébrale, notamment l'insuffisance énergétique, le flux d'ions calcium, la génération de radicaux libres et l'atténuation de l'excitotoxicité. Ces mécanismes nocifs ont été ciblés dans des modèles expérimentaux par des 
interventions pharmacologiques. Cependant, aucun de ces traitements pharmacologiques prometteurs ne s'est traduit par un usage clinique. De tels traitements « faciles d'administration » pourraient être placés sur les « chariots de code » pour être administrés au patient une fois la réanimation cardiaque réussie. En revanche, certains traitements «physiologiques", comme par exemple l'hypothermie thérapeutique, ont eu des résultats positifs aussi bien dans des contextes expérimentaux que cliniques. ${ }^{1}$ Ce type de traitement est difficile et exige une infrastructure et des ressources importantes, ainsi que beaucoup de personnel. Cependant, il est possible que même l'hypothermie thérapeutique ne soit pas indiquée pour toutes les populations humaines. ${ }^{2}$

Le pré-conditionnement est une stratégie impliquant l'augmentation de la tolérance du cerveau à l'ischémie en exposant le cerveau à des lésions ischémiques mineures ou à des agents pharmacologiques avant la survenue d'un accident ischémique majeur. Bien que, dans des études précliniques, le pré-conditionnement anesthésique se soit révélé prometteur pour protéger le cerveau contre les accidents ischémiques, il n'existe à ce jour aucune épidémiologie clinique convaincante appuyant cette pratique. ${ }^{3}$ La science préclinique au sujet du potentiel du préconditionnement anesthésique est loin d'être claire. Cette situation a été illustrée par Codaccioni et coll., qui rapportent dans un article récent une amélioration provisoire mais non soutenue des tests neurocognitifs dans un modèle d'ischémie cérébrale focale chez le rat. En vérité, cela pourrait simplement refléter et/ou être spécifique au modèle qu'ils emploient dans leurs expériences. Tout effet potentiellement neuroprotecteur du pré-conditionnement anesthésique dépend de l'agent anesthésique utilisé, de l'espèce à l'étude, du modèle d'ischémie, de l'âge et du sexe de l'animal, de la durée de pré-conditionnement, et de l'intervalle entre le préconditionnement et la lésion ischémique. ${ }^{3}$ En d'autres mots, nous ne sommes pas certains que ce type de traitement pourra être converti en un traitement qui puisse être appliqué universellement pour traiter la tGCI.

Le pré-conditionnement anesthésique est l'une des stratégies qui prépare le cerveau à anticiper ou à réagir à la lésion ischémique. Parmi les autres stratégies utilisées pour pré-conditionner le cerveau, citons les toxines, la température, les agents pharmaceutiques, et l'ischémie, cette dernière étant la stratégie la plus prometteuse. ${ }^{4,5}$ Toutes ces stratégies ont pour objectif de déséquilibrer plusieurs programmes clés de transcription du signal cellulaire afin de favoriser la survie cellulaire plutôt qu'une voie métabolique ou une protéine cible unique.

La mort neuronale après une tGCI est complexe. Des lésions caractéristiques dans les régions vulnérables du cerveau (comme l'hippocampe et le striatum) apparaissent entre 12 heures et trois jours après la réanimation et continuent d'évoluer au cours des semaines suivantes, voire des mois suivants. La plupart des cellules qui meurent le font par mort cellulaire programmée plutôt que par nécrose. Ce type de mort neuronale partage plusieurs caractéristiques avec l'apoptose, la famille de protéases à cystéine dirigées par l'aspartate (les caspases) jouant un rôle important d'exécuteurs de la mort cellulaire programmée. ${ }^{6}$ Les caspases existent normalement dans un état inactivé (sous forme de pro-caspases), mais lors de conditions de stress, une segmentation (activation) des caspases initiatrices (caspase-8 et 9) a lieu. Les caspases initiatrices peuvent être activées par des stimuli extrinsèques ou intrinsèques à la cellule. Par exemple, lorsque des cytokines en circulation telles que IL- $1 \beta$ ou TNF- $\alpha$ sont libérées après la reperfusion et se lient à des complexes de protéines transmembranaires appelés « domaines de mort », la segmentation de la caspase- 8 survient. ${ }^{7}$ Le stress cellulaire provenant d'une espèce d'oxygène réactif ou d'un flux d'ions calcium peut causer de façon intrinsèque l'activation de cascades de protéines en déstabilisant la membrane mitochondriale, générant ainsi la segmentation de la caspase-9. Une fois segmentées, les caspases initiatrices facilitent la segmentation des caspases effectrices (caspase-3), lesquelles provoquent la protéolyse de substrats cellulaires stratégiques, causant ainsi la mort contrôlée de la cellule.

Cette explication quelque peu simpliste de la mort cellulaire après ischémie ne rend pas justice à la diversité de morts cellulaires programmées qui ont été observées, ni aux nombreuses couches de contrôle métabolique, aux autres voies possibles, ou à la signalisation protéinique complexe qui a lieu entre la membrane cellulaire, les membranes mitochondriales et le noyau par l'intermédiaire des protéines de mort cellulaire. En effet, nombre de protéines pro-aptototiques clés telles que les caspases et le facteur inducteur d'apoptose (AIF) ont deux fonctions tout aussi importantes pour le fonctionnement normal de la cellule. Par conséquent, pour qu'une intervention neuroprotectrice réussisse, il faut créer et maintenir un déséquilibre entre les programmes de transduction et de transcription du signal qui favorisent la survie neuronale. En outre, une telle intervention ne devrait pas provoquer de conséquences involontaires comme l'apparition d'un cancer.

Il a été suggéré d'utiliser des agents anesthésiques en tant qu'agents neuroprotecteurs en raison de leur capacité à réduire le métabolisme cérébral. En revanche, les agents anesthésiques intraveineux, locaux et volatils préviennent la phosphorylation oxydative et la synthèse d'ATP dans les mitochondries en renversant la structure et/ou le fonctionnement des éléments présents dans la membrane intérieure des mitochondries. ${ }^{8}$ Bien que cet état semble de prime abord être neutre d'un point de vue énergétique, l'incapacité de produire des désactivateurs de l'espèce d'oxygène réactif ou 
de réguler l'homéostasie calcique intracellulaire pourrait faire pencher la balance en faveur de la mort cellulaire. ${ }^{9}$ En outre, la quantité considérable de publications récentes rapportant que les agents anesthésiques provoquent l'apoptose dans les cerveaux en développement ${ }^{10}$ et possiblement dans les cerveaux vieillissants ${ }^{11}$ pourrait changer radicalement la façon dont nous percevons les outils de notre spécialité. À l'avenir, il se peut que nous choisissions nos agents neuro-anesthésiques en fonction $\mathrm{du}$ «moindre mal » plutôt que de la « prévention de tout mal ». Cela étant dit, notre compréhension actuelle des interactions moléculaires de l'anesthésie avec les processus métaboliques dans un contexte de maladie coexistante ou de physiologie modifiée ne constitue probablement que la partie émergée de l'iceberg. En effet, un rôle neuroprotecteur clair n'a pas encore été mis en lumière.

Récemment, la tGCI a été considérée comme une «boîte noire » clinique et thérapeutique et ce, pour plusieurs raisons. Premièrement, les facteurs spécifiques au patient (par ex., âge, sexe, co-morbidité) et la cause physiopathologique sous-jacente de l'ischémie cérébrale ainsi que son traitement rendent malaisée l'évaluation de la gravité de la lésion cérébrale. Deuxièmement, avant la disponibilité de l'imagerie de diffusion et la spectroscopie par résonance magnétique, il était difficile d'établir le lien entre une lésion ischémique et l'apparition subséquente de lésions cérébrales caractéristiques. Troisièmement, il n'existe aucune option de traitement pharmacologique autre que les soins de soutien. Toutes ces limites ont fortement entravé les progrès dans ce domaine - jusqu'à ce que le potentiel de l'hypothermie thérapeutique soit rapporté. Désormais, voici ce que nous avons à notre disposition : des équipes médicales d'urgence rationalisées pour prendre en charge le déclin physiologique à l'hôpital, un fonds de recherche sur l'efficacité des méthodes de réanimation et, parfois, la possibilité d'une circulation extracorporelle en cas d'arrêt cardiorespiratoire. Dès lors, il est encore plus urgent de disposer d'un traitement complémentaire ou alternatif à l'hypothermie pour les soins neurologiques prodigués aux patients ayant subi une tGCI.

À l'avenir, les stratégies provoquant une réaction de préconditionnement (protectrice) pourraient être utilisées pour réduire l'ampleur de la lésion cérébrale associée à l'ischémie globale (c.-à-d. l'arrêt pré-circulatoire). Des données expérimentales montrent que des stimuli nociceptifs courts et mesurés de façon optimale selon un événement ischémique standard provoquent une régulation à la hausse des programmes de transduction du signal «potentiellement protecteurs », y compris la voie Akt/P13-K, les protéines kinases activées par mitogène, les canaux de potassium sensibles à l'ATP, et le NF- $\kappa \mathrm{B} .{ }^{12}$ Toutefois, il est peu probable que ces recherches transforment en fin de compte le neuroprotecteur intraveineux mentionné plus haut. L'approfondissement des recherches dans ce domaine nous aidera à limiter les mécanismes clés et les contextes spécifiques au patient liés à la survie neuronale après une tGCI. Le temps venu, nous disposerons de l'intervention « la mieux adaptée ».

Competing interests None declared.

\section{References}

1. Hypothermia after Cardiac Arrest Study Group. Mild therapeutic hypothermia to improve the neurologic outcome after cardiac arrest. N Engl J Med 2002; 346: 549-56.

2. Doherty DR, Parshuram CS, Gaboury I, et al. Hypothermia therapy after pediatric cardiac arrest. Circulation 2009; 119: 1492-500.

3. Wang L, Traystman RJ, Murphy SJ. Inhalational anesthetics as preconditioning agents in ischemic brain. Curr Opin Pharmacol 2008; 8: 104-10.

4. Miyawaki T, Mashiko T, Ofengeim D, et al. Ischemic preconditioning blocks BAD translocation, Bcl-xL cleavage, and large channel activity in mitochondria of postischemic hippocampal neurons. Proc Natl Acad Sci USA 2008; 105: 4892-7.

5. Walsh SR, Tang TY, Sadat U, Gaunt ME. Remote ischemic preconditioning in major vascular surgery. J Vasc Surg 2009; 49: 240-3.

6. Cao G, Pei W, Lan J, et al. Caspase-activated DNase/DNA fragmentation factor 40 mediates apoptotic DNA fragmentation in transient cerebral ischemia and in neuronal cultures. J Neurosci 2001; 21: 4678-90.

7. Ashkenazi A, Dixit VM. Apoptosis control by death and decoy receptors. Curr Opin Cell Biol 1999; 11: 255-60.

8. Muravchick S, Levy RJ. Clinical implications of mitochondrial dysfunction. Anesthesiology 2006; 105: 819-37.

9. Emerit J, Edeas $M$, Bricaire $F$. Neurodegenerative diseases and oxidative stress. Biomed Pharmacother 2004; 58: 39-46.

10. Mellon RD, Simone AF, Rappaport BA. Use of anesthetic agents in neonates and young children. Anesth Analg 2007; 104: 509-20.

11. Jevtovic-Todorovic V, Carter $L B$. The anesthetics nitrous oxide and ketamine are more neurotoxic to old than to young rat brain. Neurobiol Aging 2005; 26: 947-56.

12. Nakka VP, Gusain A, Mehta SL, Raghubir R. Molecular mechanisms of apoptosis in cerebral ischemia: multiple neuroprotective opportunities. Mol Neurobiol 2008; 37: 7-38. 